\title{
A new European cancer risk assessment tool for application after nuclear accidents
}

\author{
L. Walsh ${ }^{1, *}$, A. Ulanowski ${ }^{2,3}$, J.C. Kaiser ${ }^{2}$, C. Woda ${ }^{2}$ and W. Raskob ${ }^{4}$ \\ ${ }^{1}$ UZ - University of Zürich, Department of Physics, Science Faculty, Zürich, Switzerland. \\ ${ }^{2}$ HMGU - Helmholtz Zentrum München - German Research Center for Environmental Health, Neuherberg, Germany. \\ ${ }^{3}$ IAEA - International Atomic Energy Agency, IAEA Environment Laboratories, Seibersdorf, Austria. \\ ${ }^{4}$ KIT - Karlsruhe Institute of Technology, Eggenstein-Leopoldshafen, Germany.
}

\begin{abstract}
The development and application of new European software for cancer risk assessment after radiation exposure from a nuclear accident is described here. This software computes lifetime risks for several types of cancer and is intended to provide information for consideration by decision makers in the urgent and transition phases of nuclear emergencies. Such information on radiation related cancer risks can be useful to consider in the identification of protective measures (e.g., sheltering, evacuation, allocation of individuals to screening programmes and advice to take thyroid protection medication) in a differential way (i.e., for particularly susceptible sub-groups of a population). A description and application of the software for converting dose limits applicable after nuclear accidents into corresponding cancer risks, based on German population data, has already been published. Therefore, only a brief description of the main features of the software and some new illustrative results based on Swiss population data are given here, with some additional information on the input and output specification of the software.
\end{abstract}

Keywords: nuclear accidents / risk assessment / cancer / CONFIDENCE

\section{Introduction}

Radiation related cancer risk assessment software was not previously available for use immediately after a nuclear accident. For example, after the Fukushima nuclear accident (Janssens, 2013) on 11th March 2011, two years elapsed between accident occurrence and the publication of the World Health Organization (WHO) health risk assessment (WHO, 2013) report. This two-year period, after the event, was required for assessing doses, developing a risk assessment framework and developing the risk assessment software, but did not allow enough time to include a full explicit mathematical treatment of risk uncertainties. Lessons learned after Fukushima (Walsh, 2016) have emphasized the need to close such potential future time gaps between accidents and health risk assessments. Therefore, funding was provided by the European Union-CONFIDENCE (COping with uNcertainties For Improved modelling and DEcision making in Nuclear emergenCiEs) project to develop health risk assessment (HRA) software (the EU-CONFIDENCE software tool) designed to be immediately available after a nuclear accident. The tool is based on the HRA methodological framework for assessing cancer risks after the Fukushima accident as developed and recommended by a WHO expert group

\footnotetext{
* Corresponding author: linda.walsh@uzh.ch
}

(WHO, 2013; Walsh et al., 2014) and by the German ProZES project group (Jacob et al., 2017; Ulanowski et al., 2016).

This WHO Fukushima methodology has been applied here to calculate the risks of all solid cancers and leukaemia per unit relevant organ dose from contemporary models of radiation risk and for an illustrative modern European population i.e., the example calculations have been performed using the demographic data and disease statistics for Switzerland.

An important feature of the tool is a full mathematical treatment of uncertainties in the calculated risks, so that the risks can include confidence intervals. Although Swiss population data are considered here, the software tool also incorporates data for four Nordic countries and Germany and can be extended for other countries.

\section{Methods}

The methods are fully presented by (Walsh et al., 2019) and therefore only a brief description is given here. Models for additive and multiplicative excess risks in the same cancer site groupings applied by the WHO (2013) were applied to calculate lifetime risks attributable to organ/tissue doses from radiation exposures after a nuclear accident (Tab. 1). The conventional lifetime attributable risk (LAR) (Thomas et al., 1992; Vaeth and Pierce, 1990) was applied. 
Table 1. Cancer incidence outcomes included in the new CONFIDENCE software.

Cancer groupings applied and source of additive and multiplicative excess risk to dose response models

Organ dose type required

All solid cancers (ICD10:C00-C80)

Colon

Japanese A-bomb Life Span Study (LSS)

Cohort:1958-2009 (Grant et al., 2017)

All leukemia (ICD10:C91-C95), excluding CLL (ICD10:C91.1, C91.4) and ATL (ICD10:C91.5)

LSS cohort:1958-2001 (Hsu et al., 2013)

Thyroid cancer (ICD10:C73)

LSS cohort:1958-1999 (Jacob et al., 2014)

Female breast cancer (ICD10:C50)

Pooled study of eight cohorts (Preston et al., 2002)

Red bone marrow

Thyroid

Breast

Switzerland 2014 https://www.bfs,ch

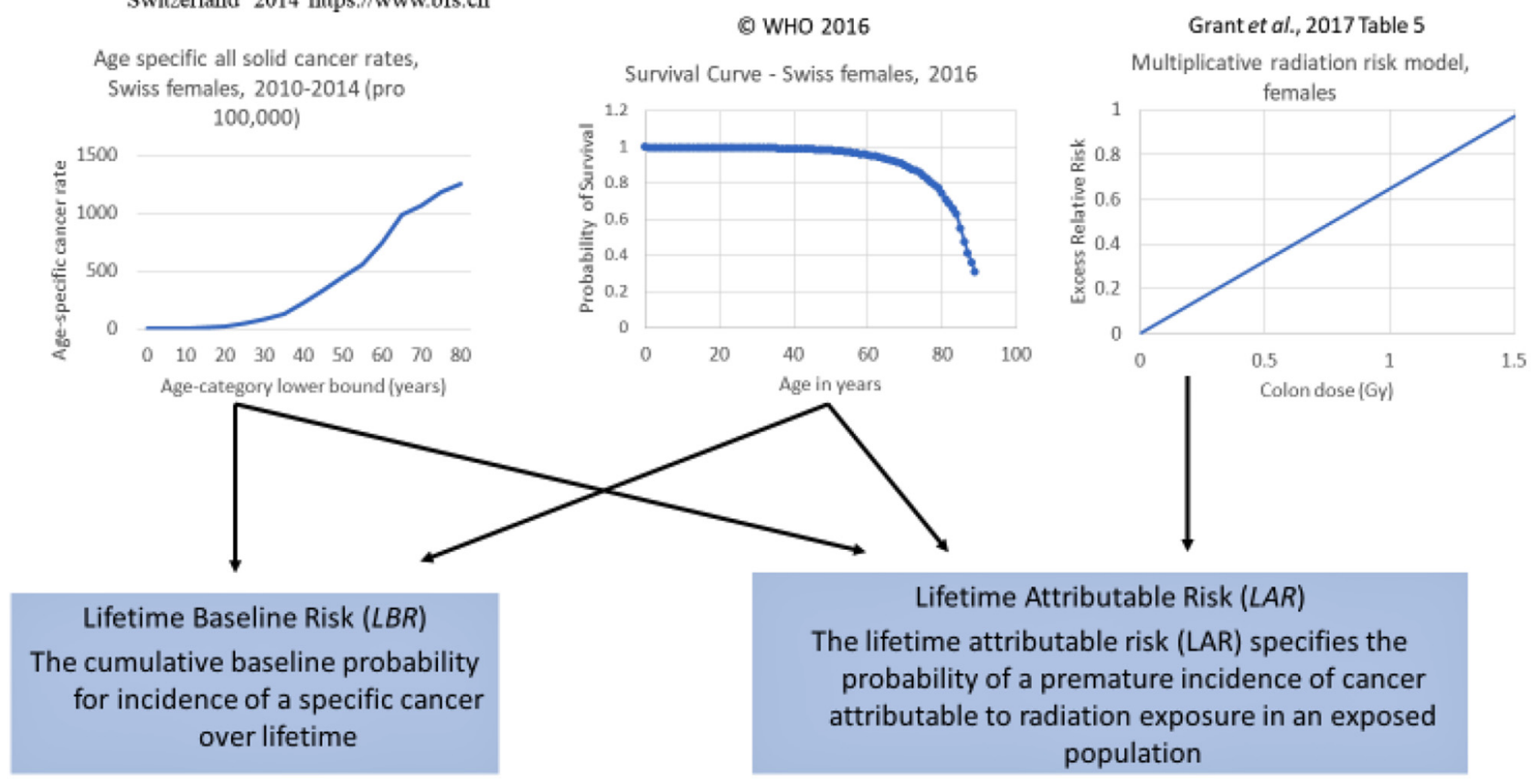

Fig. 1. Schematic diagram giving the definitions of Lifetime Baseline Risk (LBR) and Lifetime Attributable Risk (LAR) of cancer. Requirements for calculating these two quantities, for the all solid cancer grouping and Swiss females, are shown in a simplified schematic representation at the top of the diagram. The requirements, from left to right, are: age specific cancer incidence rates for 2010-2014 in Switzerland (bfs.ch); Swiss survival curves for 2016 (calculated with data from www.who.org); and radiation dose response curves for the excess relative risk as a function of colon dose (Gy) from the most recent analysis of cancer incidence in the life span study (LSS) of Hiroshima and Nagasaki A-bomb survivors (with risk parameters taken from Table 5 of Grant et al., 2017).

A schematic diagram giving the definition of LAR and examples of the input data required for calculating LAR are given in Figure 1.

Calculations of LAR are associated with large uncertainties that were quantified in this tool by applying stochastic simulations with a methodology previously described (Ulanowski et al., 2016; Jacob et al., 2014).

The following uncertainties were included:

- the radiation risk model parameters from the excess risk models (given in Tab. 1) were sampled from a multivariate normal distribution using best estimates of all the model fit parameters, including those for the model baseline, and parameter covariance matrices;
- a factor for apportioning additive and multiplicative radiation risk contributions was sampled from a uniform distribution;

- dose rate effects were sampled from a lognormal distribution with a geometric mean of 1.0;

- the minimum latency periods were sampled from a sigmoid distribution;

- uncertainties in age specific cancer incidence rates were sampled from Poisson distributions;

- the doses were sampled from a normal distribution (here, but the tool allows a choice of other distribution forms), with means of $100 \mathrm{mGy}$ organ doses appropriate to the cancer outcome type considered, Tab. 1). 

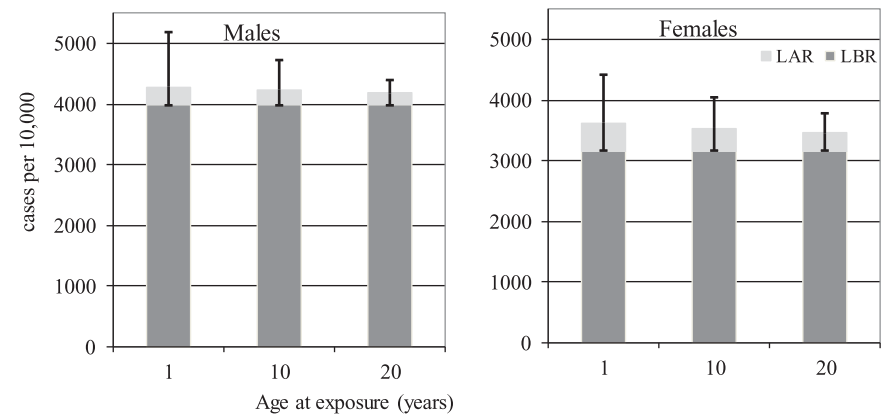

Fig. 2. Male and female all solid cancer baseline (dark grey) and radiation (light grey with error bars) risks in cases per 10,000 persons calculated from LBR and the LAR for $100 \mathrm{mGy}$ mean colon dose (normally distributed, $\sigma=50 \mathrm{mGy}$ ). Based on Swiss population data and error bars for $95 \%$ confidence intervals.
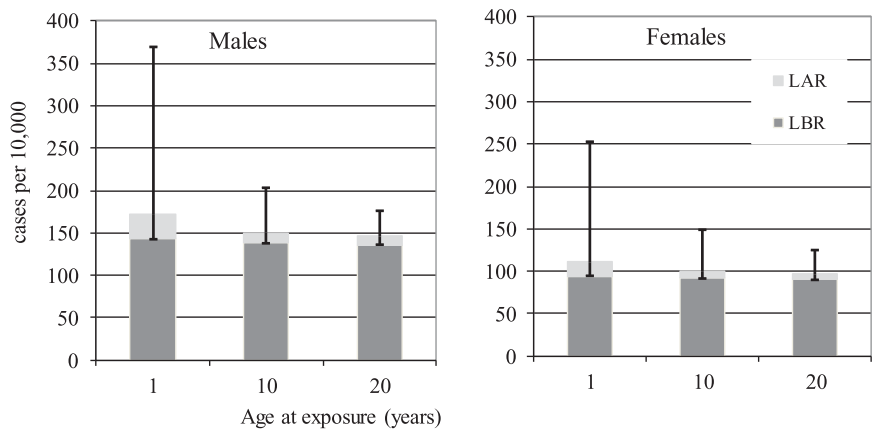

Fig. 3. Male and female leukaemia baseline (dark grey) and radiation (light grey with error bars) risks in cases per 10,000 persons calculated from LBR and the LAR for $100 \mathrm{mGy}$ mean red bone marrow dose (normally distributed, $\sigma=50 \mathrm{mGy}$ ). Based on Swiss population data and error bars for $95 \%$ confidence intervals.

Table 2. An example of the input and output format of the CONFIDENCE Tool (developed by A. Ulanowski).

Input:-

Outcome $=$ ASC $($ all solid cancer $)$

Country $=$ Switzerland

Gender $=$ Male

Age-at-exposure $=1$ (years)

Dose distribution: normal, $\mu=0.1 \mathrm{~Gy}, \sigma=0.05 \mathrm{~Gy}$

Output:-

\begin{tabular}{|c|c|c|c|c|}
\hline \multirow[t]{3}{*}{ Time (year) } & \multicolumn{4}{|l|}{ Time-integrated risk (\%) } \\
\hline & $\mathrm{BR}_{\text {time }}$ or LBR (baseline) & & $\mathrm{AR}_{\text {time }}$ or LAR (radiation-attributable) & \\
\hline & Mean & Median $(95 \% \mathrm{CI})$ & Mean & Median $(95 \% \mathrm{CI})$ \\
\hline 10 & 0.04 & $0.03(0.00-0.13)$ & 0.00 & $0.00(0.00-0.01)$ \\
\hline 50 & 2.59 & $2.56(1.88-3.52)$ & 1.06 & $0.48(0.01-5.21)$ \\
\hline Lifetime & 39.90 & $39.83(35.64-44.70)$ & 3.80 & $2.99(0.08-12.18)$ \\
\hline
\end{tabular}

LBR: Lifetime Baseline Risk (also given as baseline risk at different times since exposure, $\mathrm{BR}_{\text {time}}$ ); LAR: Lifetime Attributable Risk (also given as attributable risk at different times since exposure, $\mathrm{AR}_{\text {time }}$ ).

Radiation-related cancer risks, in terms of LAR, were estimated for both males and females initially exposed as infants (age 1 year), children (age 10 years) or adults (age 20 years) applying demographic and health statistics data from a contemporary illustrative European population (Swiss population, WHO and www.krebs.bfs.admin.ch). Table 2 gives an example of the input and output specification of the software.

\section{Results}

The LAR and lifetime baseline risk (LBR) have been simply converted from probabilities (Tab. 2) to numbers of excess cases and number of baseline cases per 10,000 persons, for age at exposure 1, 10 and 20 years, and are given in
Figures 2 and 3, for all solid cancers and leukaemia, respectively.

The results for all solid cancers (Fig. 2) show that females have a larger radiation risk than males over a lifetime, but males have a larger lifetime baseline risk than females. The grouping "all solid cancer" will provide risk estimates with a higher statistical power than is obtainable for individual cancer sites, due to the much larger number of all solid cancers compared to the numbers of site specific cancers, in the datasets used to fit the radiation dose response curves for the additive and multiplicative excess risk to dose response models (obtained in the studies cited in Tab. 1), see Figure 1. However, it is noteworthy that the $95 \%$ confidence intervals on the numbers of all solid cancer cases expected per 10,000 persons, at $100 \mathrm{mGy}$ colon dose, over lifetime are still large e.g., 462 (95\%CI: 12; 1258), 375 (95\%CI: 8; 879) and 294 (95\%CI: 6; 
631) for females exposed as infants, children and adults, respectively.

Figure 3 gives the results for leukaemia. Here, the radiation risk sex differences, reflect those differences reported in the additive and multiplicative A-bomb life span study risk models (Hsu et al., 2013) i.e., the multiplicative model did not support a gender effect but the additive model did, with a female to male ratio of 0.66 . Due to the equal average probabilities of additive and multiplicative transfer types in the LAR calculations applied here, it can be seen that the male radiation lifetime risks are consistently slightly larger than the female risks at the same doses and for all ages at exposure considered. The male leukaemia baseline risks over a lifetime are also consistently larger than the female risks for all three ages at exposure considered.

\section{Discussion and conclusions}

Given the time lapse between the Fukushima accident and the development of HRA software mentioned in the introduction, there is great potential for risk assessment tools that have been fully developed and are ready for operation, before any nuclear accident actually takes place. However, in adopting a "WHO" framework and developing software for use in health risk assessment, it is important to avoid misunderstandings when interpreting lifetime attributable risks, LAR, as presented here. Although LAR values can be based on individual (but not person specific) doses, they cannot represent an individual's cancer risks because there is generally no information on important co-factors that influence a particular individual's cancer risk (e.g., individual radiation sensitivity; genetic pre-disposition to cancer and lifestyle factors). Furthermore, the Swiss population-based incidence and survival curves, used in the LAR calculations, only represent average values for the national population considered at one point in time. LAR should therefore be interpreted as an average risk for specific ages at exposure and genders. The number of baseline and radiation related cases observed in the future will include other sources of variability (uncertainty), dependent on several factors including the size of the population group, and future developments in the secular trends in population statistics (on which the risks are based), which are not accounted for in the software HRA tool. Further work has also been done on methods that reduce the dependence, of radiation related risk assessments on population statistics and survival curves (Ulanowski et al., 2019). Initial work has also been undertaken to assess the usefulness of directly integrated the risk assessment tool into currently available decision support systems (e.g., JRODOS, Ehrhardt and Weis, 2000) and this is a topic for future developments.

Acknowledgement. The work described in this paper was conducted within the CONFIDENCE project, which was part of the CONCERT project. This project has received funding from the Euratom research and training programme 20142018 under grant agreement No. 662287.

Disclaimer (Art. $29.5 \mathrm{GA}$ ). This publication reflects only the author's view. Responsibility for the information and views expressed therein lies entirely with the authors. The European Commission is not responsible for any use that may be made of the information it contains.
This paper makes use of data obtained from the Radiation Effects Research Foundation (RERF), Hiroshima and Nagasaki, Japan. RERF is a public interest incorporated foundation funded by the Japanese Ministry of Health, Labour and Welfare (MHLW) and the U.S. Department of Energy (DOE). The data include information obtained from the Hiroshima City, Hiroshima Prefecture, Nagasaki City, and Nagasaki Prefecture Tumor Registries and the Hiroshima and Nagasaki Tissue Registries. The conclusions in this paper are those of the authors and do not necessarily reflect the scientific judgment of RERF or its funding agencies.

\section{References}

Ehrhardt J, Weis A. (Eds.). 2000. RODOS: decision support system for off-site nuclear emergency management in Europe, final report of the RODOS project. Brussels: European Commission. Report EUR 19144, ISBN No. 92-828-9773-7, includes 2 CDs with all the technical details of the RODOS Systems.

Grant EJ, Brenner A, Sugiyama H, Sakata R, Sadakane A, Utada M, Cahoon EK, Milder CM, Soda M, Cullings HM, Preston DL, Mabuchi K, Ozasa K. 2017. Solid cancer incidence among the life span study of atomic bomb survivors: 1958-2009. Radiat. Res. 187: 513-537.

Hsu WL, Preston DL, Soda M, Sugiyama H, Funamoto S, Kodama K, Kimura A, Kamada N, Dohy H, Tomonaga M, Iwanaga M, Miyazaki Y, Cullings H, Suyama A, Ozasa K, Shore R, Mabuchi K. 2013. The incidence of leukemia, lymphoma and multiple myeloma among atomic bomb survivors: 1950-2001. Radiat. Res. 179: 361-382.

Jacob P, Kaiser C, Ulanovsky A. 2017. ProZES - a tool for assessment of assigned share of radiation in probability of cancer development. Bundesamt für Strahlenschutz (BfS). Report BfSRESFOR-120/17. Available from http://doris.bfs.de/jspui/handle/ urn:nbn:de:0221-2017032014253.

Jacob P, Kaiser JC, Ulanovsky A. 2014. Ultrasonographic surveys and thyroid cancer in the Fukushima Prefecture. Radiat. Environ. Biophys. 53: 291-401 and 403 (erratum).

Janssens A. 2013. EU basic safety standards and the European response to the Fukushima accident. Radioprotection 48: S19-S26.

Preston DL, Mattsson A, Holmberg E, Shore R, Hildreth NGM, Boice JD. 2002. Radiation effects on breast cancer risk: a pooled analysis of eight cohorts. Radiat. Res. 158: 220-235.

Thomas D, Darby S, Fagnani F, Hubert P, Vaeth M, Weiss K. 1992. Definition and estimation of lifetime detriment from radiation exposures: principles and methods. Health Phys. 63: 259-272.

Vaeth M, Pierce DA. 1990. Calculating excess lifetime risk in relative risk models. Environ. Health Prospect. 87: 83-94.

Ulanowski A, Kaiser JC, Schneider U, Walsh L. 2019. On prognostic estimates of radiation risk in medicine and radiation protection. Radiat. Environ. Biophys. 58: 305-319.

Ulanowski A, Eidemüller M, Güthlin D, Kaiser JC, Shemiakina E, Jacob P. 2016. ProZES: a tool for assessment of assigned share of radiation in probability of cancer development (part II). Bundesamt für Strahlenschutz (BfS). Report BfS-RESFOR-116/ 16. Availiable from http://doris.bfs.de/jspui/handle/urn:nbn: de:0221-2016112214169.

WHO. 2013. Health risk assessment from the nuclear accident after the 2011 Great East Japan Earthquake and Tsunami based on a preliminary dose estimation. WHO. Available from http://www. who.int/ionizing_radiation/pub_meet/fukushima_risk_assess ment_2013/en. 
Walsh L. 2016. Fukushima health risk assessment: lessons learned. In: EU Scientific Seminar 2014 Fukushima - Lessons learned and issues, Luxembourg, 18 November 2014, European Commission publication.

Walsh L, Zhang W, Shore RE, Auvinen A, Laurier D, Wakeford R, Jacob P, Gent N, Anspaugh LR, Schüz J, Kesminiene A, van Deventer E, Tritscher A, del Rosario Pérez M. 2014. A framework for estimating radiation-related cancer risks in Japan from the 2011 Fukushima nuclear accident. Radiat. Res. 182: $556-572$.

Walsh L, Ulanowski A, Kaiser JC, Woda C, Raskob W. 2019. Risk bases can complement dose bases for implementing and optimizing a radiological protection strategy in urgent and transition emergency phases. Radiat. Environ. Biophys. 58: 539-552.

Cite this article as: Walsh L, Ulanowski A, Kaiser JC, Woda C, Raskob W. 2020. A new European cancer risk assessment tool for application after nuclear accidents. Radioprotection 55(HS1): S95-S99 